\title{
SARS-CoV-2 viremia is associated with distinct proteomic pathways and predicts COVID-19 outcomes
}

Yijia Li, ${ }^{1,2}$ Alexis M. Schneider, ${ }^{3,4}$ Arnav Mehta, ${ }^{2,3,5,6}$ Moshe Sade-Feldman, ${ }^{2,3,6}$ Kyle R. Kays, ${ }^{2}$ Matteo Gentili, ${ }^{3}$ Nicole C. Charland, ${ }^{2}$ Anna L.K. Gonye, ${ }^{2,3,6}$ Irena Gushterova, ${ }^{2,3,6}$ Hargun K. Khanna, ${ }^{2}$ Thomas J. LaSalle, ${ }^{2,3,6}$ Kendall M. Lavin-Parsons, ${ }^{2}$ Brendan M. Lilley, ${ }^{2}$ Carl L. Lodenstein, ${ }^{2}$ Kasidet Manakongtreecheep, ${ }^{2,3}$ Justin D. Margolin, ${ }^{2}$ Brenna N. McKaig, ${ }^{2}$ Blair A. Parry, ${ }^{2}$ Maricarmen Rojas-Lopez, ${ }^{7,8}$ Brian C. Russo, ${ }^{7,8}$ Nihaarika Sharma, ${ }^{2,3}$ Jessica Tantivit, ${ }^{2,3,9}$ Molly F. Thomas, ${ }^{2,3,9}$ James Regan, ${ }^{1}$ James P. Flynn, ${ }^{1}$ Alexandra-Chloé Villani, ${ }^{2,3,9}$ Nir Hacohen, ${ }^{2,3,6}$ Marcia B. Goldberg, ${ }^{2,3,7,8}$ Michael R. Filbin, ${ }^{2,3}$ and Jonathan Z. Li ${ }^{1}$

'Brigham and Women's Hospital and ${ }^{2}$ Massachusetts General Hospital, Harvard Medical School, Boston, Massachusetts, USA. ${ }^{3}$ Broad Institute of MIT and Harvard, Cambridge, Massachusetts, USA. ${ }^{4}$ Department of Biological Engineering, Massachusetts Institute of Technology, Cambridge, Massachusetts, USA. ${ }^{5}$ Dana-Farber Cancer Institute, Harvard Medical School, Boston, Massachusetts, USA. ${ }^{6}$ Center for Cancer Research, Department of Medicine, and 'Center for Bacterial Pathogenesis, Division of Infectious Diseases, Department of Medicine, Massachusetts General Hospital, Boston, Massachusetts, USA. ${ }^{8}$ Department of Microbiology, Harvard Medical School, Boston, Massachusetts, USA. ${ }^{9}$ Center for Immunology and Inflammatory Diseases, Department of Medicine, Massachusetts General Hospital, Boston, Massachusetts, USA

BACKCROUND. SARS-CoV-2 plasma viremia has been associated with severe disease and death in COVID-19 in small-scale cohort studies. The mechanisms behind this association remain elusive.

METHODS. We evaluated the relationship between SARS-CoV-2 viremia, disease outcome, and inflammatory and proteomic profiles in a cohort of COVID-19 emergency department participants. SARS-CoV-2 viral load was measured using a quantitative reverse transcription PCR-based platform. Proteomic data were generated with Proximity Extension Assay using the Olink platform.

RESULTS. This study included 300 participants with nucleic acid test-confirmed COVID-19. Plasma SARS-CoV-2 viremia levels at the time of presentation predicted adverse disease outcomes, with an adjusted $\mathrm{OR}$ of $10.6(95 \% \mathrm{Cl} 4.4-25.5, P<0.001)$ for severe disease (mechanical ventilation and/or 28-day mortality) and 3.9 (95\% Cl 1.5-10.1, $P=0.006$ ) for 28-day mortality. Proteomic analyses revealed prominent proteomic pathways associated with SARS-CoV-2 viremia, including upregulation of SARS-CoV-2 entry factors (ACE2, CTSL, FURIN), heightened markers of tissue damage to the lungs, gastrointestinal tract, and endothelium/vasculature, and alterations in coagulation pathways.

CONCLUSION. These results highlight the cascade of vascular and tissue damage associated with SARS-CoV-2 plasma viremia that underlies its ability to predict COVID-19 disease outcomes.

FUNDING. Mark and Lisa Schwartz; the National Institutes of Health (U19AI082630); the American Lung Association; the Executive Committee on Research at Massachusetts General Hospital; the Chan Zuckerberg Initiative; Arthur, Sandra, and Sarah Irving for the David P. Ryan, MD, Endowed Chair in Cancer Research; an EMBO Long-Term Fellowship (ALTF 486-2018); a Cancer Research Institute/Bristol Myers Squibb Fellowship (CRI2993); the Harvard Catalyst/Harvard Clinical and Translational Science Center (National Center for Advancing Translational Sciences, NIH awards UL1TR001102 and UL1TR002541-01); and by the Harvard University Center for AIDS Research (National Institute of Allergy and Infectious Diseases, 5P30AI060354).

\section{Introduction}

With coronavirus disease 2019 (COVID-19) causing over 2 million deaths globally by early 2021 (1), there remains an urgent need to

Authorship note: NCC, ALKG, IG, HKK, TJL, KMLP, BML, CLL, KM, JDM, BNM, BAP, MRL, BCR, NS, JT, and MFT contributed equally to this work.

Conflict of interest: The authors have declared that no conflict of interest exists. Role of funding source: The funders had no role in study design, data collection, data analysis, or preparation of the manuscript.

Copyright: @ 2021, American Society for Clinical Investigation.

Submitted: February 10, 2021; Accepted: May 6, 2021; Published: July 1, 2021.

Reference information: / Clin Invest. 2021;131(13):e148635.

https://doi.org/10.1172/JCl148635. elucidate disease pathogenesis to improve clinical management and treatment. There is increasing evidence that COVID-19, caused by severe acute respiratory syndrome coronavirus 2 (SARS-CoV-2), frequently manifests pathology beyond the pulmonary tract (2-4). In both immunocompromised and immunocompetent hosts, SARS-CoV-2 nucleic acids have been detected across a broad range of extrapulmonary sites, including spleen, heart, liver, and intestinal tract (5-9). In addition, endothelial cells are known to express ACE2, and some reports have suggested that direct infection of endothelial cells may be leading to a hypercoagulable state with vascular and downstream organ damage. Furthermore, viremia has been implicated in transplacental transmis- 


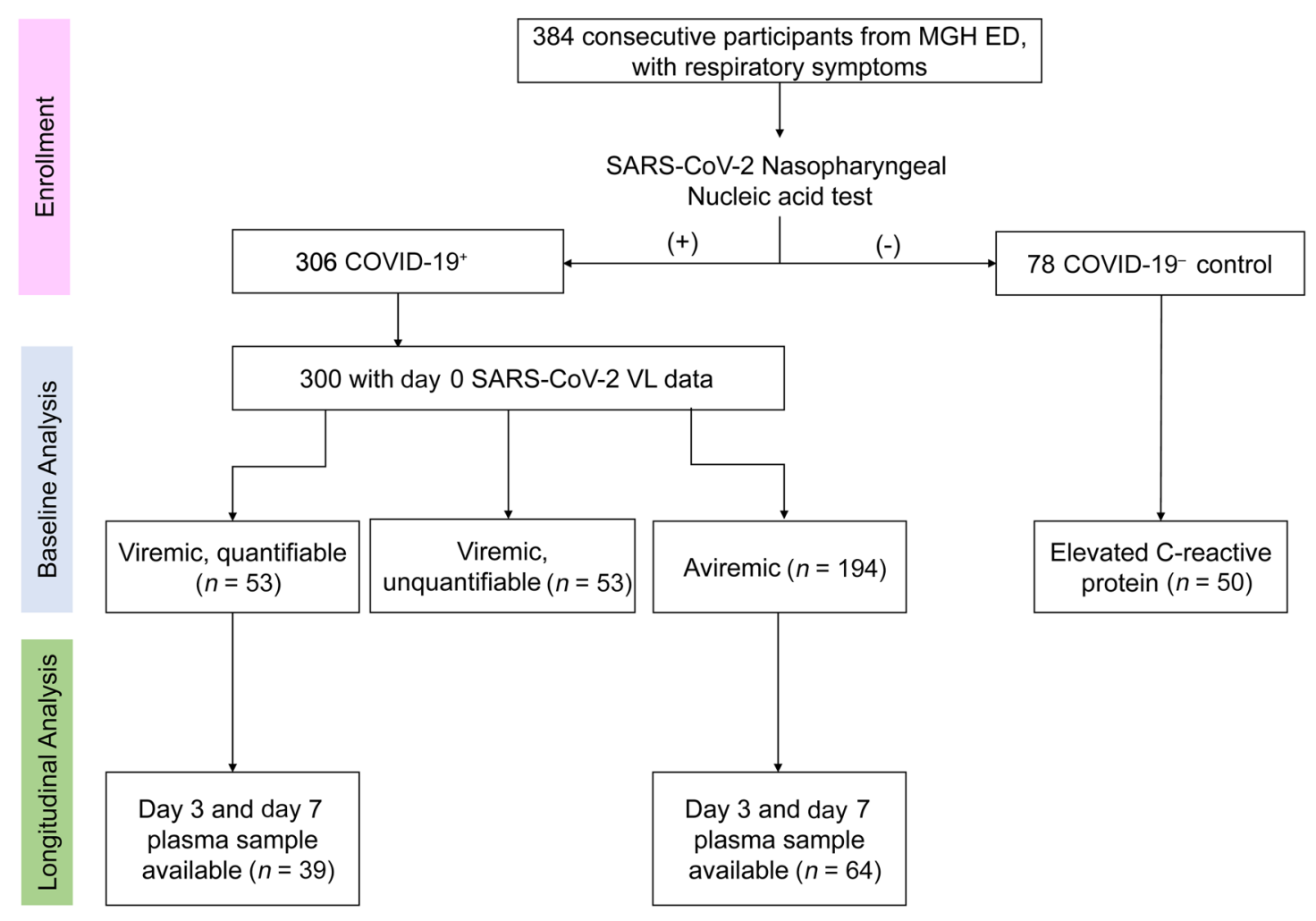

Figure 1. Enrollment and follow-up flow diagram. MCH, Massachusetts General Hospital; ED, emergency department; VL, viral load.

sion $(7,10)$. These reports suggest that dissemination of infection outside of the respiratory tract into the circulatory system may be a critical step for COVID-19 pathogenesis.

We and others have previously demonstrated that SARSCoV-2 plasma viremia in hospitalized patients is associated with severe disease and death (11-14). However, these studies have been limited by sampling late during the disease course and relatively small sample sizes. Here, we performed plasma SARSCoV-2 viral load quantification and proteomic analysis and assessed neutralizing antibody titers in a large cohort of emergency department (ED) patients enrolled at the time of initial presentation. We evaluated whether levels of SARS-CoV-2 viremia could predict COVID-19 disease outcomes after adjustment for multiple potential confounders. We also performed proteomic analysis to reveal prominent pathways that are upregulated in the setting of plasma viremia and determined the relationship between plasma SARS-CoV-2 viral load and levels of neutralizing antibodies.

\section{Results}

Baseline participant characteristics. This cohort consisted of 306 participants with a molecular diagnosis of COVID-19, of whom 300 had successful plasma SARS-CoV-2 viral load quantification and thus were included in this current analysis (Figure 1). Baseline characteristics were reported in our prior study (15) and are summarized in Table 1. Thirty-nine percent of participants were at least 65 years old, and about half of participants were female. Eleven percent of participants had morbid obesity (BMI $\geq 40 \mathrm{~kg} /$ $\left.\mathrm{m}^{2}\right), 47 \%$ had a diagnosis of hypertension, and $36 \%$ had diabetes. Fifty-three of 300 participants (18\%; Figure $2 \mathrm{~A}$ ) had a base- line SARS-CoV-2 viral load above the limit of quantification (2 $\log _{10}$ copies/mL). Individuals with quantifiable SARS-CoV-2 viral load at the time of ED presentation were older, had higher rates of diabetes, and had clinical laboratory values consistent with higher disease severity, including lower lymphocyte count and higher creatinine, C-reactive protein (CRP), and troponin (Table 1). Median time between symptom onset and ED presentation was 7 days (IQR 4-11) and was comparable between individuals with viral load above and below the limit of quantification (Figure 2B and Supplemental Figure 1; supplemental material available online with this article; https://doi.org/10.1172/JCI148635DS1). Quantified SARS-CoV-2 viral load at the time of ED presentation was correlated with older age; lower lymphocyte count; higher inflammatory markers, including CRP, D-dimer, and lactate dehydrogenase; and both renal and liver dysfunction (Figure $2 \mathrm{C}$ ).

$S A R S-C o V-2$ viremia at the time of ED presentation predicted adverse clinical outcomes during the hospitalization. Elevated SARS$\mathrm{CoV}-2$ viremia $\geq 2 \log _{10}$ copies/mL at the time of ED presentation was a strong predictor of maximal COVID-19 disease acuity within 28 days of enrollment. Those with elevated viral load were significantly more likely to have severe disease ( $82 \%$ vs. $26 \%, P$ $<0.001$; Figure 3A), which included those who died or required invasive mechanical ventilation. Participants with SARS-CoV-2 viral loads less than $2 \log _{10}$ copies/mL were further categorized into those with detectable viral load below the limit of quantification and those with undetectable viral load (aviremic). This revealed a dose-dependent effect of viremia on adverse outcomes (Figure 3B). Higher levels of SARS-CoV-2 viremia upon ED presentation were associated with increased severity at all time points 
Table 1. Summary of baseline characteristics

Total $(n=300) \quad$ Viremic within quantification range $e^{A}(n=53)$

\section{Aviremic or below quantification} range $(n=247)$

Age $(n, \%)$

$$
<50 \text { years }
$$

50-64 years

$\geq 65$ years

$\operatorname{Sex}(n, \%)$

Female

Male

Race $(n, \%)$

Non-White $(n, \%)$

White $(n, \%)$

Ethnicity $(n, \%)$

\section{Hispanic}

Non-Hispanic

Morbid obesity ${ }^{\mathrm{B}}$ (BMI $\left.\geq 40 \mathrm{~kg} / \mathrm{m}^{2}\right)(n, \%)$

Heart diseases $(n, \%)$

Lung diseases $(n, \%)$

Hypertension $(n, \%)$

Diabetes $(n, \%)$

Immunocompromised condition $(n, \%)$

Lymphopenia <1000 cells $/ \mathrm{mm}^{3}(n, \%)$

Creatinine elevation $>1.20 \mathrm{mg} / \mathrm{dL}(n, \%)$

$\mathrm{CRP}^{\mathrm{C}}>100 \mathrm{mg} / \mathrm{dL}$

D-dimer ${ }^{0}>1000 \mathrm{ng} / \mathrm{mL}(n, \%)$

Troponin elevation within 72 hours $(n, \%)$

Baseline SARS-CoV-2 viral load, log copies/mL (median, IQR)

Percentage of detectable but not quantifiable viremia $(n, \%)$

$96(32.0)$
$86(28.7)$
$118(39.3)$

$144(48.0)$

$156(52.0)$

$150(50.0)$

150 (50.0)

$162(54.0)$

$138(46.0)$

$33(11.8)$

46 (15.3)

$64(21.3)$

$143(47.7)$

$108(36.0)$

$25(8.3)$

$149(49.7)$

64 (21.3)

146 (50.7)

151 (53.2)

24 (8.0)

NA

53 (17.7)
10 (18.9)

15 (28.3)

28 (52.8)

20 (37.7)

33 (62.3)

31 (58.5)

22 (41.5)

29 (54.7)

24 (45.3)

7 (13.7)

$6(11.3)$

$6(11.3)$

28 (52.8)

28 (52.8)

$5(9.4)$

33 (62.3)

$17(32.1)$

38 (73.1)

34 (66.7)

8 (15.1)

$2.68(2.39-3.63)$

$0(0.0)$
86 (34.8)

$71(28.7)$

90 (34.4)

124 (50.2)

$123(49.8)$

119 (48.2)

$128(51.8)$

$133(53.8)$

$114(46.2)$

$26(11.4) \quad 0.19$

$40(16.2) \quad 0.37$

$58(23.5) \quad 0.05$

$115(46.6) \quad 0.41$

$80(32.4) \quad 0.005$

$20(8.10) \quad 0.75$

$47(19.0) \quad 0.04$

$108(45.8) \quad<0.001$

$117(50.2) \quad 0.03$

$16(6.5) \quad 0.04$

NA

53 (21.5)

\subsection{4}

$116(47.0) \quad 0.04$

${ }^{A}$ Quantification range for viremia is $\geq 2.0$ log copies/mL; ${ }^{\mathrm{B}} 280$ participants with available BMI; ' 288 participants with available CRP; ${ }^{\mathrm{D} 284}$ participants with available D-dimer. CRP, C-reactive protein; SARS-CoV-2, severe acute respiratory syndrome coronavirus 2; IQR, interquartile range. measured - days 0, 3, 7, and 28 (Supplemental Figure 2). Twentyeight-day mortality was $32 \%$ in the high viral load group and $9.7 \%$ in the low viral load group $(P<0.001)$. Higher plasma viral load was also consistently associated with higher risk of severe disease and death across age groups (Supplemental Figure 3).

We also assessed the impact of SARS-CoV-2 by univariate and multiple logistic regression for severe disease. Viremia $\geq 2 \log _{10}$ copies/mL had an odds ratio (OR) of 12.6 (95\% CI 6.0-26.5, $P<$ 0.001) in univariate logistic regression for severe disease (Table 2). After adjustment for other baseline variables with a $P$ value less than 0.1 in univariate analyses, viremia remained significantly associated with severe disease, with an adjusted OR (aOR) of 10.6 (95\% CI 4.4-25.5, $P<0.001$ ). Similarly, viremia $\geq 2 \log _{10}$ copies/ $\mathrm{mL}$ was strongly associated with death within 28 days (Table 2 and Supplemental Table 1), with an aOR of 3.9 (95\% CI 1.5-10.1, $P=0.006)$ in multivariate analysis. The results were consistent when viral load was categorized into 3 strata $\left(2 \log _{10}\right.$, detectable below $2 \log _{10}$, and aviremic) or analyzed as a continuous variable (Supplemental Table 2). Each $\log _{10}$ increase in viral load was associated with an aOR 2.49 of severe disease $(P<0.001)$ and aOR 1.46 of death $(P=0.01)$. Finally, higher viral load was also associated with a higher risk of death at day 28 by Cox proportional hazard modeling (adjusted hazard ratio 4.0, 95\% CI 1.9-8.7,
$P<0.001$; Supplemental Figure 4). We performed logistic regression to evaluate demographic and laboratory variables associated with SARS-CoV-2 viremia. In multivariate analysis, only diabetes and CRP greater than $100 \mathrm{mg} / \mathrm{dL}$ were associated with viremia (Supplemental Table 3).

SARS-CoV-2 viremia at the time of ED presentation was associated with diffuse tissue damage, tissue fibrosis/repair, and elevation of proinflammatory markers. We included in the proteomic analysis 247 participants with either viremia above quantification range (viremic) or undetectable viremia (aviremic). Unsupervised clustering of participants by uniform manifold approximation and projection (UMAP) using Olink proteomic results demonstrated a diffuse pattern of distribution for viremic participants, with certain enrichment in the right lower quadrant and left upper quadrant (Figure 4A). In hierarchical clustering of participants by viremia-associated protein signatures, viremic participants were dispersed into several distinct clusters, indicating the heterogeneity of proteomic signatures among viremic participants (Supplemental Figure 5). In addition, viremia and severe disease showed overlap in the proteomic signatures (Supplemental Figure 5).

To identify differentially expressed proteins between viremic and aviremic participants, we created linear models to fit each of the proteins at day 0 with viremia status as a main effect and adjust- 


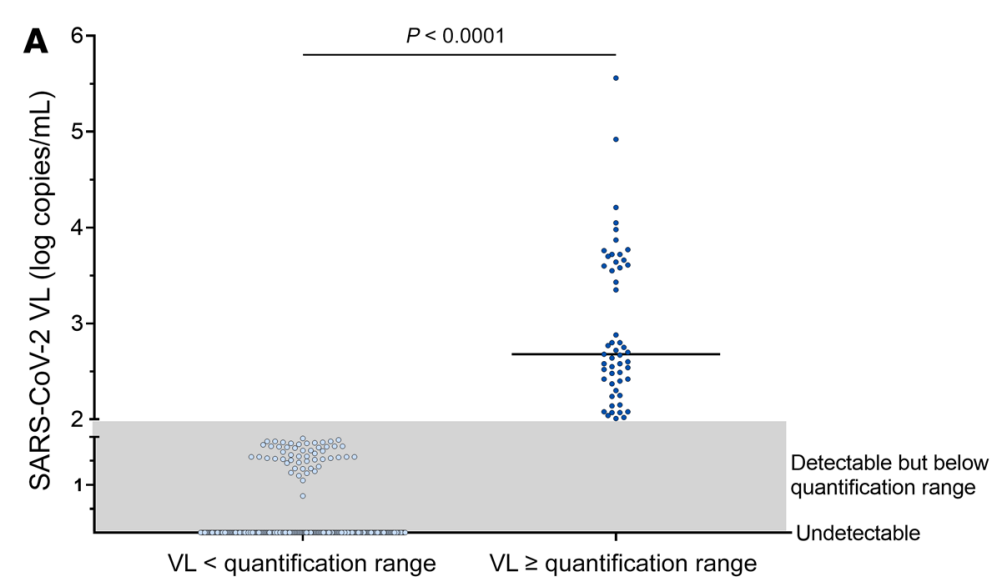

\section{B}

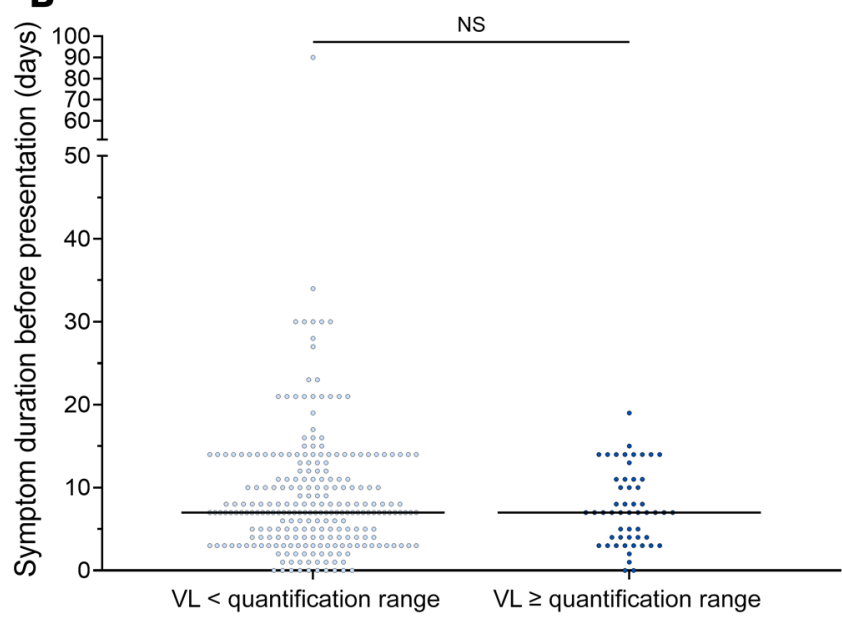

\section{C}

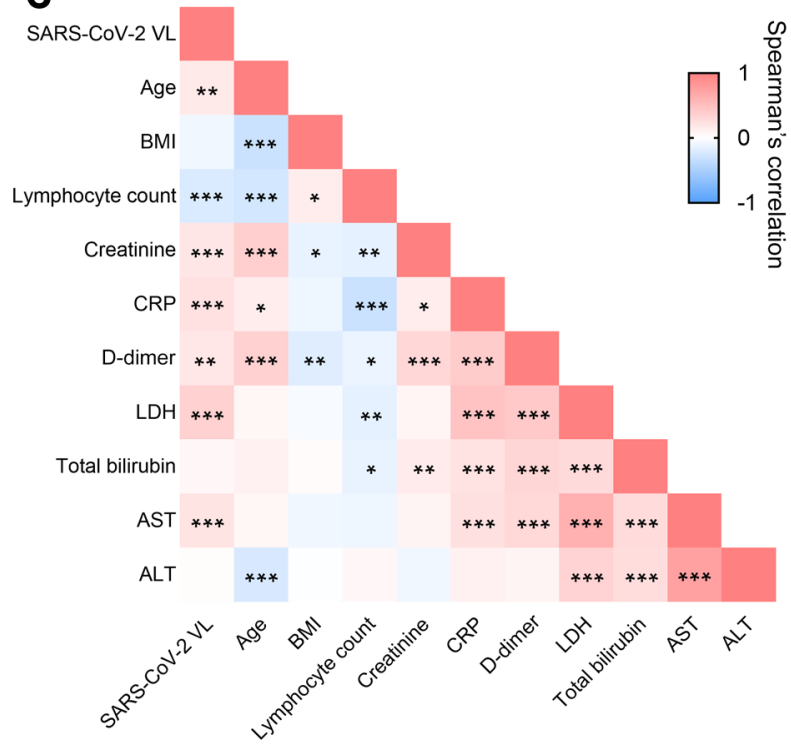

ed for age, demographics, and key comorbidities (Figure 4B). A number of prominent proteomic pathways were associated with higher plasma viral load. First, viremic participants demonstrated higher expression of viral response and interferon/monocytic pathway proteins, including IL-6, C-C motif chemokine ligand 7 (CCL7)/monocyte chemotactic protein 3 (MCP-3), CCL2O/ macrophage inflammatory protein $3 \alpha$ (MIP-3A), CXCL10/IFN- $\gamma-$
Figure 2. SARS-CoV-2 viremia at day 0. (A) Distribution of SARS-CoV-2 viral load (VL). Fifty-three participants had viremia within the quantification range with median viral load 2.68 log copies/mL; 247 participants had viral loads below the range of quantification or detection. We used the Mann-Whitney test to compare 2 groups. (B) Duration between symptom onset and ED presentation was comparable between the viremic (quantifiable) and the aviremic/ viremic (unquantifiable) group. The Mann-Whitney test was used for comparison. (C) Pairwise correlation heatmap between viral load and baseline factors (Spearman's rank correlation coefficient). $n=300 .{ }^{*} P<0.05 ;{ }^{* *} P<0.01$; ${ }^{* * *} P<0.001$. LDH, lactate dehydrogenase; AST, aspartate transaminase; ALT, alanine transaminase.

induced protein 10 (IP-10), CXCL9/monokine induced by IFN- $\gamma$ (MIG), CXCL8/IL-8, IFN- $\lambda 1$ (IFNL1), CCL2/ MCP-1, CCL19/MIP-3B, CCL3/MIP-1A, CXCL11, IL-15, and IL-18 (Figure 4C). Nicotinamide phosphoribosyl transferase (NAMPT), an important regulator upstream of IL-6 production (16), was also upregulated in the viremic group. Second, viremia was associated with elevation of tissue damage markers (17), including gastrointestinal (GI) tract/pancreas/liver markers (e.g., REG3A, REG1B, AGR2, GP2, MUC13, FABP1, PLA2G1B, PLA2G10, SPINK1, EPCAM, IGFBP1); lung markers, especially surfactant proteins (SFTPD, SFTPA1/2, AGER, LAMP3); and cardiac markers (troponin I3/TNNI3, NTproBNP, MB, CDH2). KRT18, KRT19, and RUVBL1, which are widely expressed in a variety of tissue types, including GI tract, pancreas, lungs, urinary system, and adipose tissue, are also significantly elevated in viremic participants, serving as markers of pan-tissue damage. It is worth mentioning that some of these proteins also likely play an important role in tissue fibrosis, including SERPINE1, CHI3L1, and CTSL along with TGF- $\alpha$, TGF- $\beta$, and type IV collagen proteins (COL6A3, COL4A1). Third, higher plasma viral load was associated with signs of endovascular damage, prominent endothelium/vascular markers and angiogenesis-related proteins (ANGPT2, ANGPTL4, EPO, ESM1, VEGFA, VCAM), and coagulation pathway-related markers (F3/tissue factor, SERPINE1, slight elevation of vWF, along with downregulation of PROC) (Figure 4C). In addition, we noted upregulation in viremic participants of certain complement pathway-related proteins, especially PTX3, and to a lesser degree C1QA.

After adjustment for disease severity in the models, certain proinflammatory markers (IL-6, CCL7, CXCL10/IP-10, CXCL11), pulmonary injury markers (SFTPD, SFTPA1/2, AGER), GI tract/pancreas/liver markers (AGR2, IGFBP1, PLA2G10, EPCAM, MUC13, GP2), coagulation markers (F3), tissue fibrosis markers (CHI3L1), and pan-tissue injury markers (e.g., epithelial cell proteins RUVBL1, KRT18/19) remained significantly associated with SARS-CoV-2 viremia, independent of disease severity (Figure 4B). Interestingly, we also noted elevation 

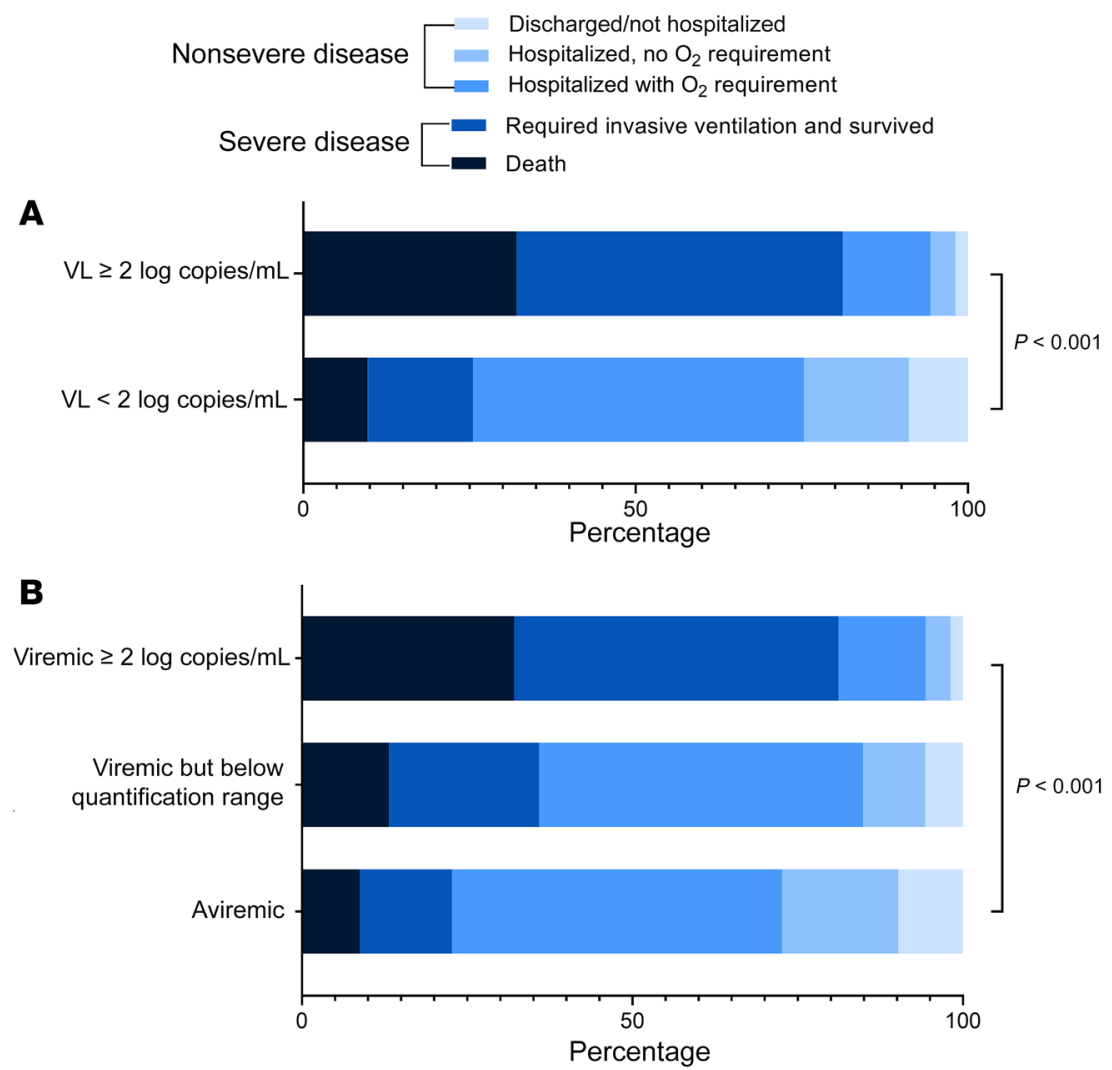

Figure 3. Association between baseline SARS-CoV-2 viral load and maximal disease severity (acuity ${ }_{\text {max }}$ ). (A) Disease severity categorized by viral load (VL) above and below the quantification limit $\left(\geq 2 \log _{10}\right.$ copies $/ \mathrm{mL}$ vs. $<2 \log _{10}$ copies $/ \mathrm{mL}$ ). (B) Disease severity categorized by viral load within the quantification range, below the quantification range but detectable, or aviremic. The $\chi^{2}$ test or Fisher's exact test was used for comparison. $n=300$.

with negative SARS-CoV-2 test as control. The majority of differentially expressed proteins from Figure 4, B and C, remained significantly different between the viremic and COVID-19-negative control groups, including key cytokines (CCL7, CXCL10/IP-10, IL-15), monocyte-related proteins (CD14, VSIG4), neutrophil-related proteins (PRTN3), lungrelated proteins (SFTPA1/A2, AGER, LAMP3), GI system-related proteins (AGR2, PLA2G10, LBP, GP2), coagulation-related proteins (F3, $\mathrm{vWF}$ ), endothelium/angiogenesis-related proteins (ESM1, ANGPT2), complement pathway proteins (C1QA, PTX3), and certain entry factors (FURIN, CTSL, NRP1) (Supplemental Figure 7). In comparison, IL-6 was comparable between the 2 groups, as a marker for monocyte-related inflammation in general.

of certain proteins that facilitate SARS-CoV-2 infection, including its receptor ACE2 (18), CD209/DC-SIGN (19), NRP1 (20, 21), and the entry facilitators/proteases FURIN (22) and cathepsin B/L (CTSB/CTSL) (ref. 23 and Figure 4C). Lactate dehydrogenase, a commonly used laboratory marker indicating tissue damage and pyroptosis (24), was highly correlated to lung-related, severity-independent markers (SFTPA1/2, AGER), especially in the viremic group (Supplemental Figure 6).

In addition to proteins related to tissue injury, fibrosis, and repair, we noted significant elevation of proteins related to certain monocytes/dendritic cells (i.e., CD14, CD163) and plasmablasts (i.e., CD138/SDC1, TXNDC5) based on publicly available RNA-sequencing databases derived from PBMCs (25-28). Certain neutrophil markers were also elevated in the viremic group, including CHI3L1, IL1RN, MMP-9, and proteinase-3 (PRTN3) (ref. 28 and Supplemental Table 4). After adjustment for severe disease, certain monocyte/dendritic cell markers and neutrophil markers remained significantly associated with viremia (Supplemental Table 4). To ensure that the SARS-CoV-2 viremia rather than generalized inflammation was associated with these differentially expressed proteins, we included 50 participants with respiratory dysfunction and an inflammatory profile (CRP $>10 \mathrm{mg} / \mathrm{dL}$ ) who presented to ED during a similar period of time
Table 2. Factors associated with severe COVID-19 and death

\begin{tabular}{|c|c|c|c|c|}
\hline & Univariate $\mathrm{OR}(95 \% \mathrm{Cl})$ & $P$ & Multivariate OR (95\% Cl) & $P$ \\
\hline \multicolumn{5}{|l|}{ Severe disease } \\
\hline \multicolumn{5}{|l|}{ SARS-CoV-2 viremia } \\
\hline Aviremic or below quantification range & Reference & & Reference & \\
\hline Viremic $\geq 2$ log copies/mL & $12.56(5.96-26.46)$ & $<0.001$ & $10.59(4.40-25.51)$ & $<0.001$ \\
\hline \multicolumn{5}{|l|}{ Death by day 28} \\
\hline \multicolumn{5}{|l|}{ SARS-CoV-2 viremia } \\
\hline Aviremic or below quantification range & Reference & & Reference & \\
\hline Viremic $\geq 2$ log copies/mL & $4.39(2.15-8.96)$ & $<0.001$ & $3.86(1.47-10.14)$ & 0.006 \\
\hline
\end{tabular}



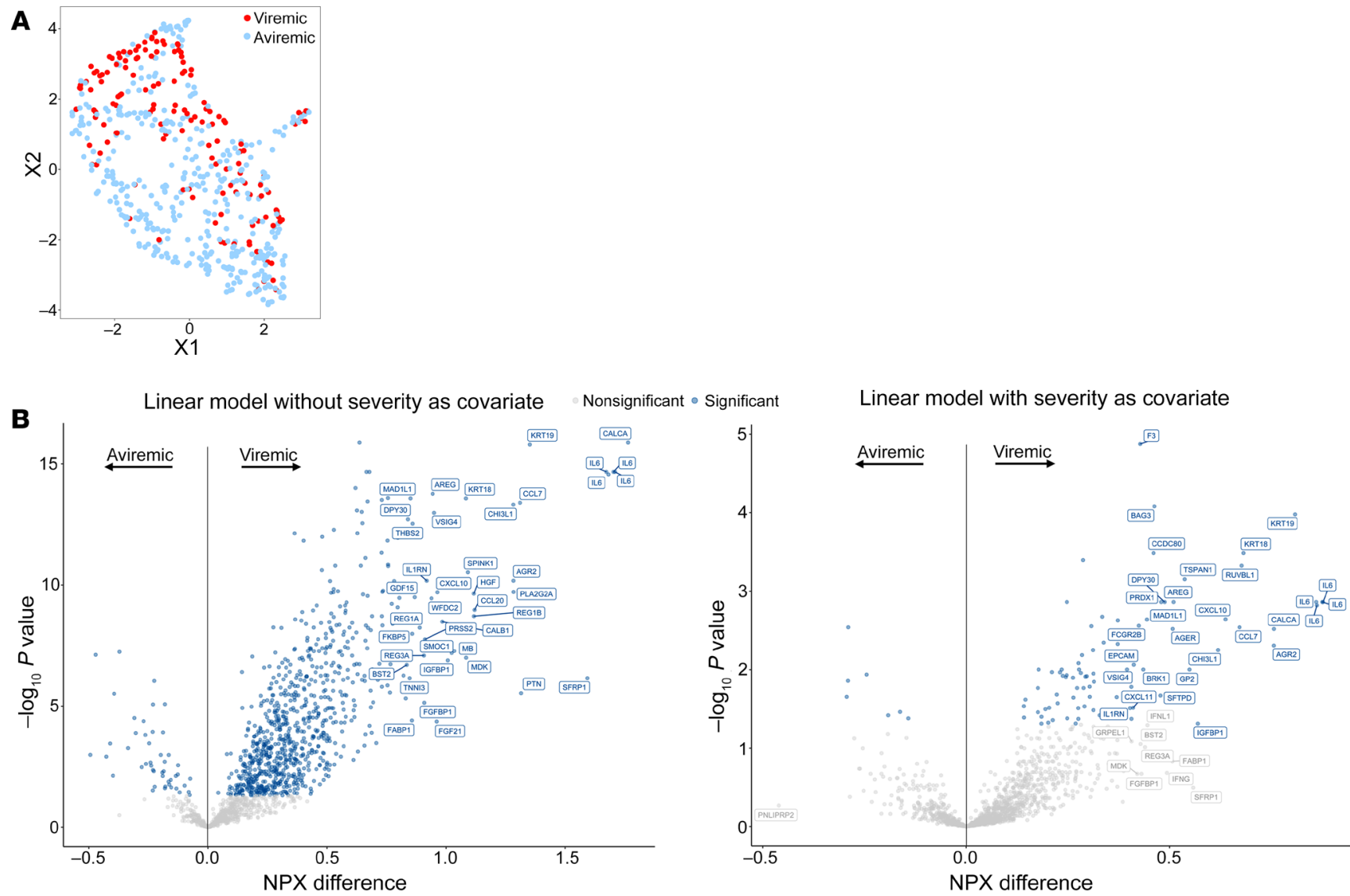

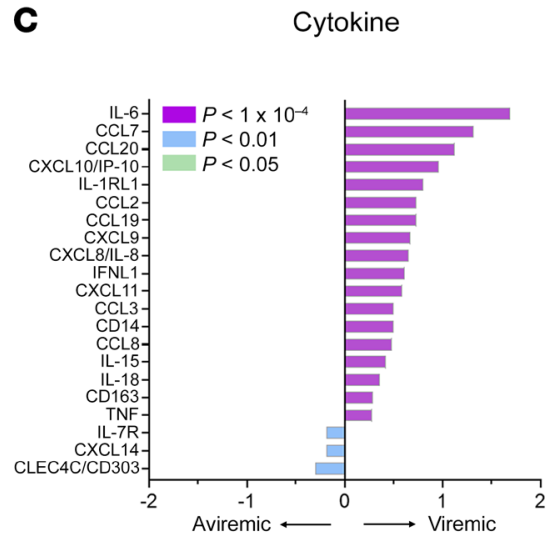

$\log _{2}$ fold change

Lung

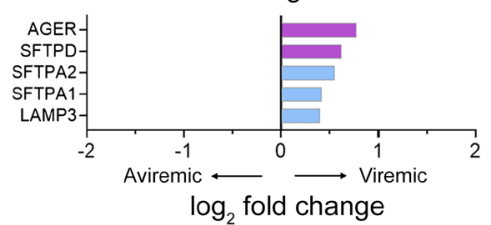

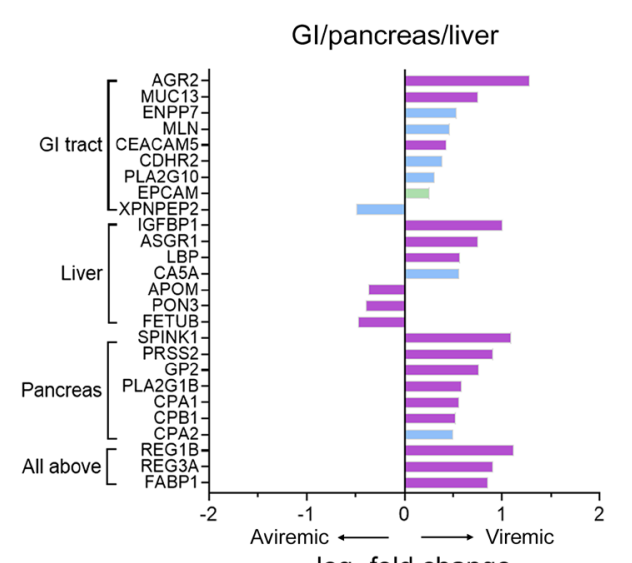

$\log _{2}$ fold change

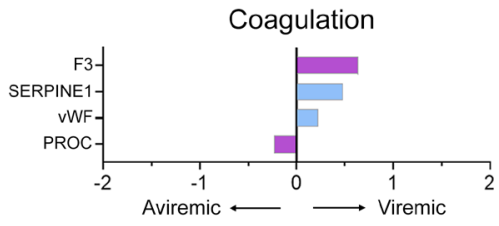

$\log _{2}$ fold change
Endothelium/angiogenesis
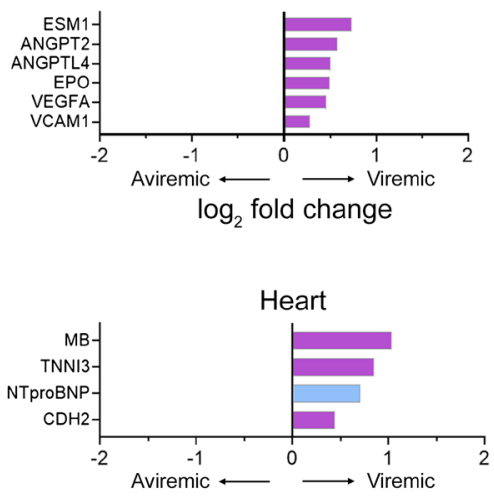

$\log _{2}$ fold change

Entry factors

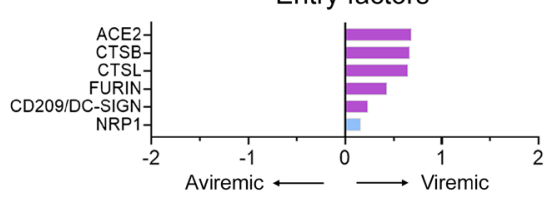

$\log _{2}$ fold change

Figure 4. Plasma proteomic biomarkers and predictors of disease severity. (A) Unsupervised clustering uniform manifold approximation and projection (UMAP) for COVID-19-positive patients at days 0, 3, and 7. Red dots indicate viremic participants, and blue dots indicate aviremic participants. (B) Volcano plots showing normalized protein expression (NPX) differences in protein levels between viremic and aviremic participants. The left panel is derived from a linear model without severity as a covariate; the right panel is derived from a linear model with severity as a covariate. (C) Representative differentially expressed proteins between viremic and aviremic participants. Adjusted $P$ values are color-coded as indicated. $n=247$. 
A

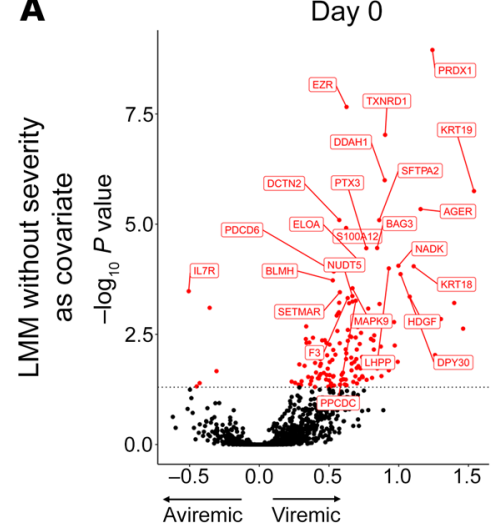

NPX difference

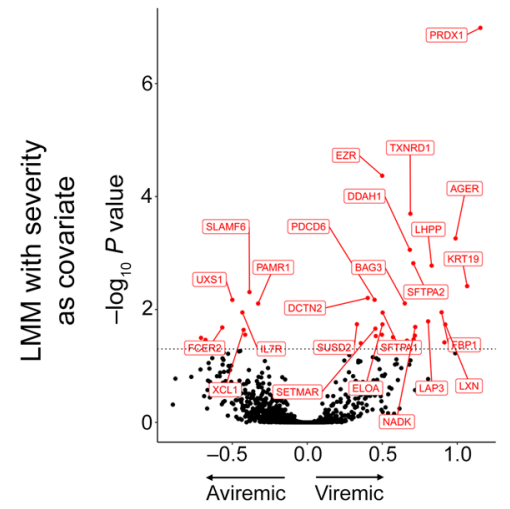

NPX difference

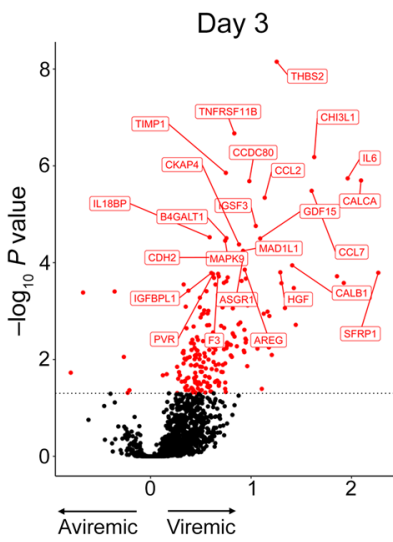

NPX difference

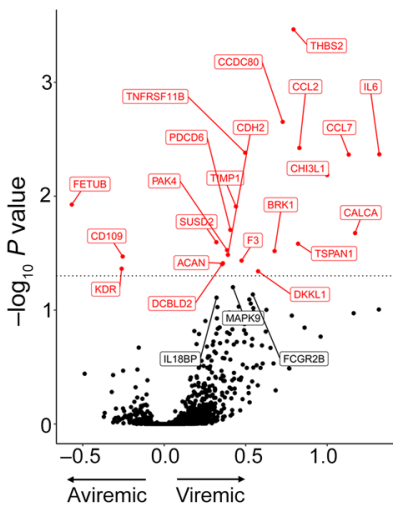

NPX difference

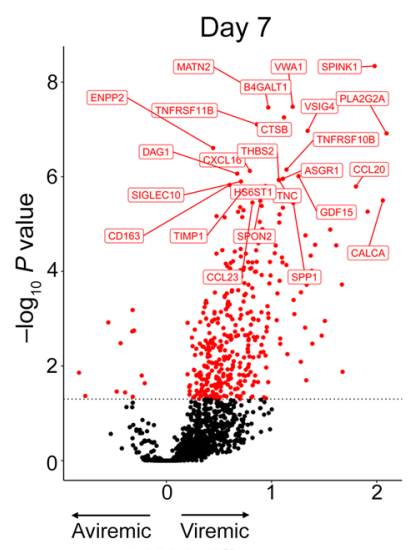

NPX difference
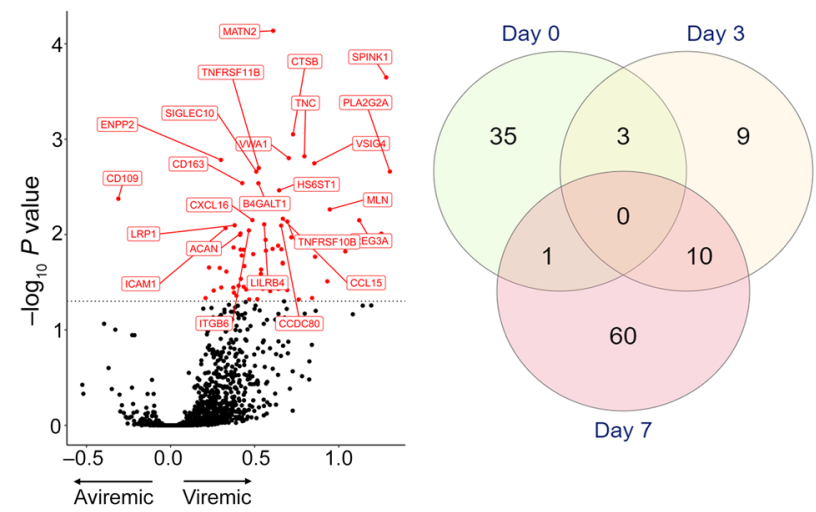

iic $\overrightarrow{\text { Viremic }}$

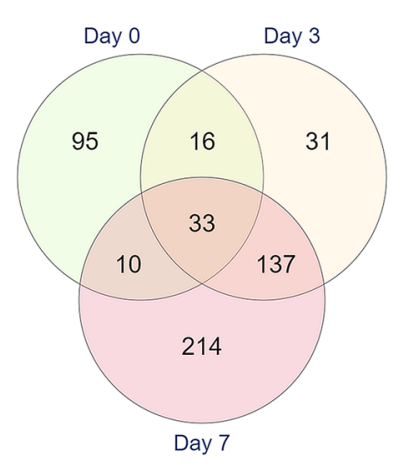

B

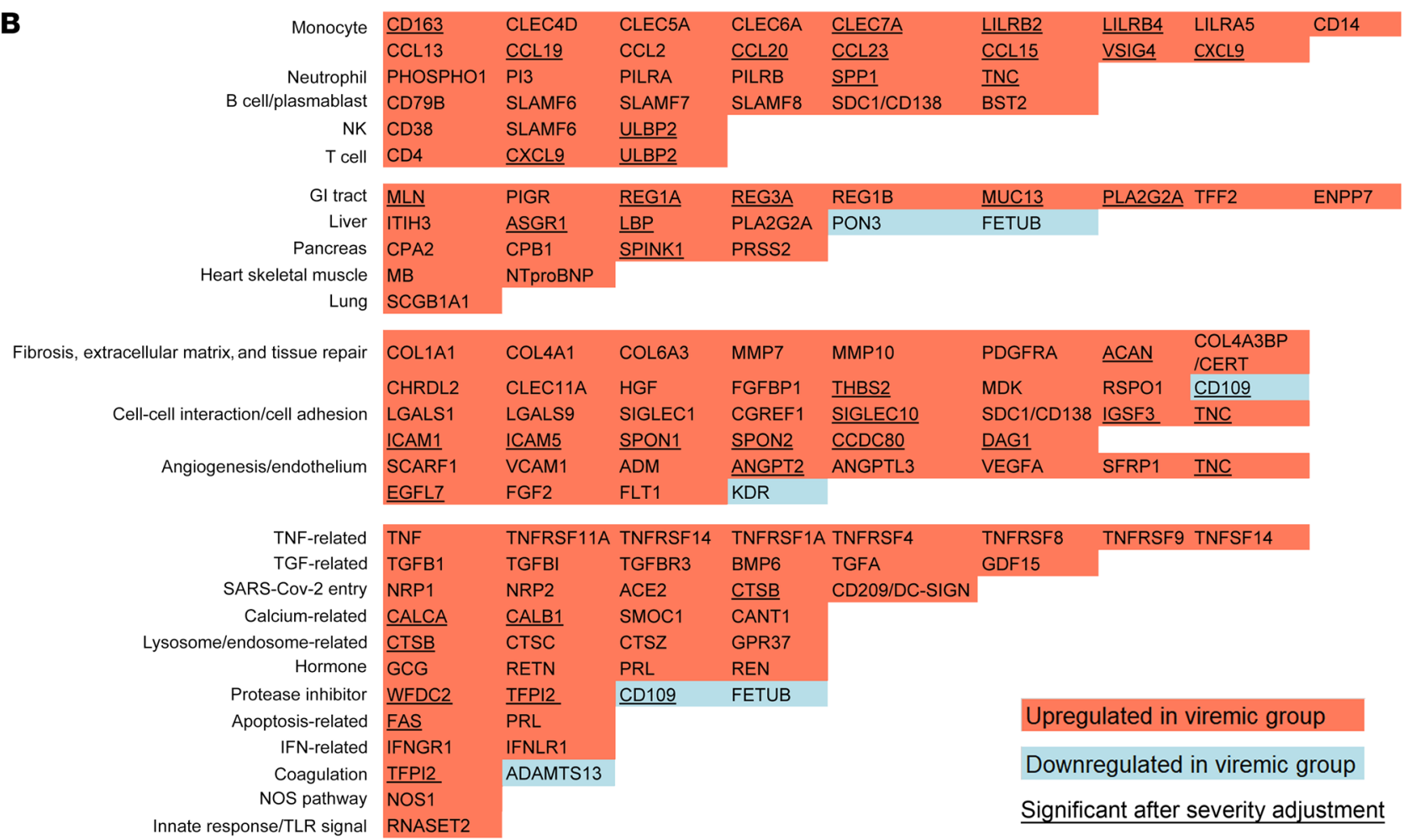

Fibrosis, extracellular matrix, and tissue repair

COL1A1

$\operatorname{cOL} 4 A 1$

NPX difference

Figure 5. Temporal trends of differentially expressed proteins between viremic and aviremic groups. (A) Volcano plots showing linear mixed model (LMM) of differentially expressed proteins at different time points ( $P$ values indicate group differences calculated by Tukey's post hoc method, $n=103$ at each time point). Venn diagrams demonstrate the overlap of differentially expressed proteins at different time points. (B) Selected proteins differentially expressed in the viremic group later in hospitalization (only at day 7 or only at day $3+$ day 7 ). Underlines indicate statistical significance after adjustment for severe disease. $n=103$. 

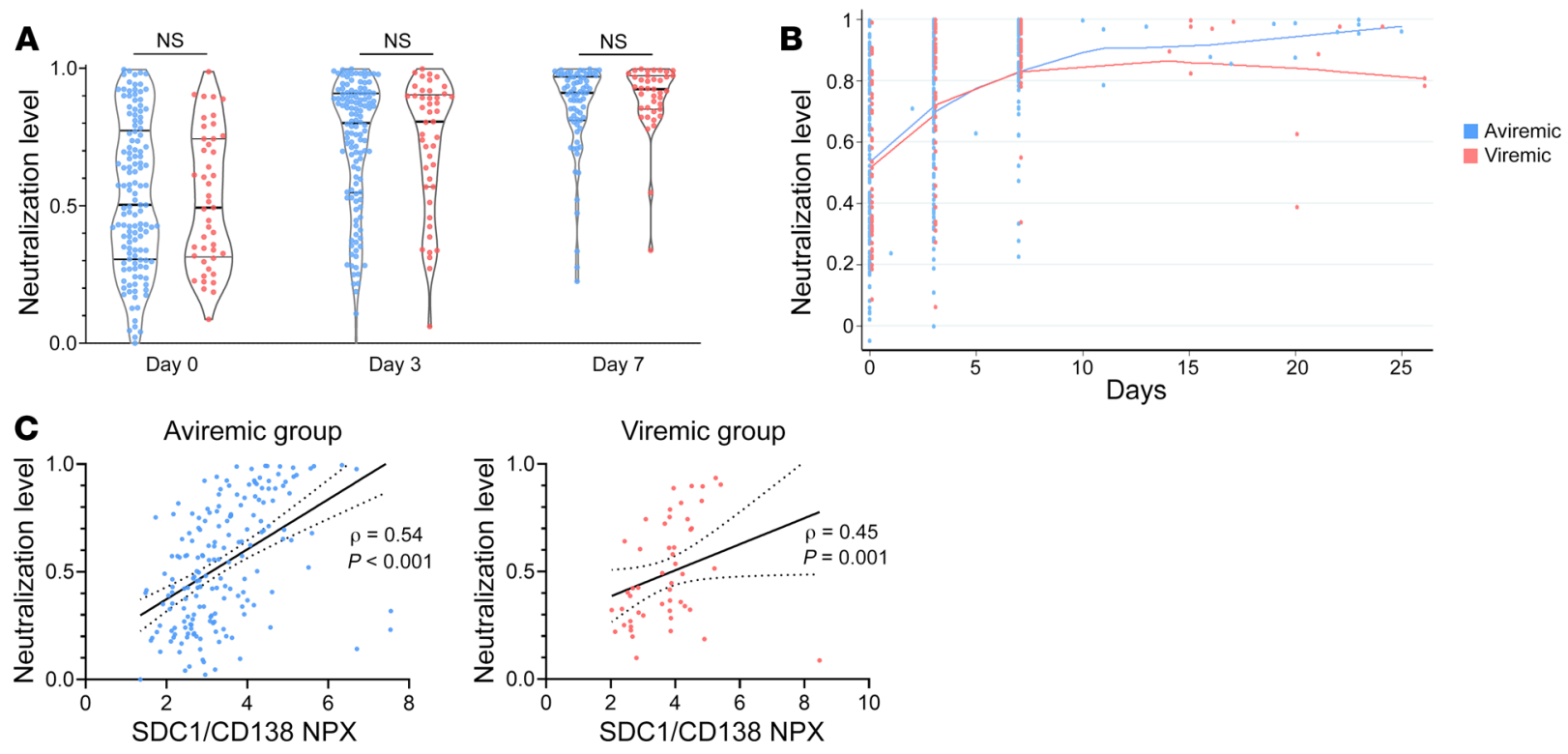

Figure 6. Neutralization level and viremia. (A) Violin plot of neutralization levels stratified by viremia status. Mann-Whitney $U$ test was used to evaluate the difference between 2 groups. (B) Neutralization rate between viremic and aviremic groups. LOWESS (locally weighted scatterplot smoothing) smooth regression was performed to depict the trajectory of neutralizing rates between 2 groups. (C) Correlation between SDC1/CD138 (a marker for plasmablasts) NPX and neutralizing rate at day 0 . Linear regression (solid line) with $95 \% \mathrm{Cls}$ (dotted lines) is shown. Spearmann's correlation was used to evaluate the correlation between SDC1/CD138 NPX and neutralizing rates. $n=175$.

teins related to tissue repair/fibroblasts; heart and skeletal muscle; GI tract; and pancreas. Finally, SFTPD, a locally secreted surfactant protein in lungs, clustered with certain apoptosis-related proteins (e.g., BAX) and housekeeping proteins located in the cytosol (NPM1, MAPK9, EIF4G1) and mitochondria (ATP5IF1, GRPEL1). Lung tissue markers, including SFTPA1/2 and AGER, were moderately correlated to upstream apoptosis-related proteins (Fas, PDCD family, and BAX/BID/BCL2L11) and weakly correlated to pyroptosis-related proteins (Supplemental Figure 9). Using elastic-net logistic regression with cross-validation, SARS-CoV-2 viremia along with day 0 proteomic data yielded good predictive performance for severe disease (AUC 0.83, 95\% CI 0.80-0.86; Supplemental Figure 10A), and Olink proteomic data yielded good predictive performance for viremia (AUC 0.81, 95\% CI 0.78-0.83; Supplemental Figure 10B).

Viremic participants experienced prolonged tissue damage, inflammation, and elevation in viral entry factors. To assess the longitudinal impact of viremia, we focused on 103 hospitalized participants with complete proteomic data from days 0,3 , and 7 (acuity level from A1 to A4). Notably, only participants with baseline viremia greater than $2 \log _{10}$ copies/mL and aviremic participants were included. We first looked at the trajectory of those proteins identified in the day 0 analysis (Figure 4). Viremic participants had persistently higher levels of proinflammatory markers beyond day 0 , especially those related to monocyte activation. For some inflammatory markers (e.g., TNF, IL-18, and CD14), differences between groups became highly divergent over time with hyper-accentuated inflammatory responses in viremic participants (Supplemental Figure 11). Longitudinal proteomic analysis also demonstrated the persistent elevation of proteomic pathways reflecting organ damage, endothelial damage, and a hypercoag- ulable state. Certain complement pathway-related proteins and entry-related factors were also persistently elevated in the viremic group (Supplemental Figure 11).

We next fit linear mixed models for each protein with time and viremia status as main effects and adjusted for age, demographics, and key comorbidities to identify proteins that were significant for the interaction between viremia and time (Figure 5A). We further noted an uptrend in monocyte-related proteins in the viremic group at later time points, followed by neutrophil- and B cell/plasmablastrelated proteins (Figure 5B). Many of these markers were significantly elevated even after adjustment for severe disease (labeled in bold font). We also noted an association between viremia and persistent, yet uptrending tissue damage levels, especially those from the GI system. Furthermore, levels of numerous proteins related to tissue fibrosis, tissue repair, and extracellular matrix began to increase at later time points, including several collagen proteins, ACAN, MDK, etc. (Figure 5B). In parallel, proteins related to endothelial damage and angiogenesis were further upregulated in the viremic group, in conjunction with a dysregulated hemostasis state featuring decreases in ADAMTS13 (Supplemental Figure 11 and Figure 5C). Certain pathways that play a significant role in severe COVID-19, especially interferon-related pathways (31), did not show significant difference between the viremic and aviremic groups during follow-up (Supplemental Figure 12).

To determine whether differences in proteomic profiles persisted at late time points between viremic and aviremic individuals, we performed a proteomic analysis on 27 participants (13 viremic, 14 aviremic) with available plasma beyond day 10 of hospitalization. In this limited population, we did not detect significant differences in the proteomic profile between participants (data not shown). 
Viremia at the time of ED presentation is not associated with neutralization levels. Finally, we evaluated the relationship between SARS-CoV-2 viremia and neutralization level. We included participants with neutralization data available at baseline and at least 1 follow-up time point. Only participants with baseline viremia greater than $2 \log _{10}$ copies/mL and aviremic participants were included. Neutralization levels between the viremic and aviremic groups were not significantly different at days 0, 3, and 7 (Figure 6A). In the subset of participants with neutralization data available beyond day 7 , no clear difference was observed between the viremic and aviremic groups (Figure 6B). At the time of ED presentation, levels of SDC1/CD138, a cardinal and specific marker for plasmablasts $(26,28)$, were significantly correlated with neutralization level, irrespective of the presence of viremia (Figure 6C). We also conducted an analysis including a subgroup of participants with available viral load at day $3(n=49)$ and day $7(n=$ 39). Undetectable viral load at day 3 or day 7 was not associated with higher neutralizing antibody titers (Supplemental Figure 13).

\section{Discussion}

In this study, we report a comprehensive analysis of SARS-CoV-2 viremia and its associations with disease outcomes and proteomic pathways from a cohort of ED patients with COVID-19. To our knowledge, this is the largest longitudinal cohort to explore this topic. The results demonstrate that SARS-CoV-2 plasma viremia at the time of ED presentation predicts maximal COVID-19 disease severity and mortality within 28 days. In addition, for the first time to our knowledge, we uncovered proteomic signatures upregulated in the setting of SARS-CoV-2 viremia, including prominent pathways highlighting lung and systemic tissue damage, tissue fibrosis and repair, a pronounced proinflammatory response, perturbed hemostasis, and upregulation of viral entry factors.

It is now clear that SARS-CoV-2 infection extends outside the respiratory system (2), and the detection of plasma viremia represents the "link" for extrapulmonary multiorgan involvement and adverse outcomes. Systemic invasion from the respiratory tract is not unique to SARS-CoV-2, as viremia has also been described for other respiratory viruses including SARS-CoV-1 (32), influenza virus (33), respiratory syncytial virus (34), and adenovirus (35). We and others have previously demonstrated that SARS-CoV-2 viremia is more commonly detected in critically ill populations $(11,12,14,36)$, and is correlated with cardinal proinflammatory markers, including IL-6 (11, 14), IP-10/ CXCL10 (37), CCL2/MCP-1 (37), and markers of endothelial damage (37). These studies were limited by a lack of true viral load quantification, small sample sizes that could not account for confounders, and/or the evaluation of hospitalized patients only late in their disease course. Here, we report the largest study to date of plasma SARS-CoV-2 viremia using a quantitative viral load assay that allowed for the confirmation of the previous findings $(11,14)$ even after adjustment of multiple potential confounding variables. A particular strength of our study was the ability to enroll all acutely ill patients upon ED arrival and thereby minimize selection bias. Our results demonstrate that at the time of ED presentation, plasma SARS-CoV-2 viral load levels independently predicted, in a dose-dependent manner, severe disease and death within the next 28 days. SARS-CoV-2 viremia was associated with clinical markers associated with disease severity, including elevated CRP and lymphopenia.

Our proteomic analysis represents another strength of this study, which demonstrates unique pathways in patients with plasma viremia that together orchestrate a "perfect storm." Viremic individuals displayed a proteomic pattern of broad tissue damage, highlighted by severe lung damage, GI damage, persistent proinflammatory marker elevation, endovascular damage, and tissue fibrosis. While previous studies have reported the elevation of certain nonspecific tissue damage markers in viremic individuals, especially lactate dehydrogenase $(37,38)$, our study allows a far more precise evaluation and demonstrates that respiratory tract and GI tract/liver/pancreas injury constitutes some of the major contributors to tissue injury in patients with SARS-CoV-2 viremia. Our proteomic analysis extends the results of a proteomic evaluation of an autopsy tissue study (39) by showing that many of the pathways of tissue and endothelial cell damage can already be identified relatively early in the disease course and may be mediated by systemic dissemination of SARS-CoV-2 infection.

We observed the upregulation of a panel of angiogenesis- and endothelial damage-related markers in viremic patients. In addition, several key factors in the coagulation pathway, including factor III (F3), von Willebrand factor (vWF), and SERPINE1 (plasmin inhibitor), were elevated in the viremic group, in conjunction with a decrease in ADAMTS13, a metalloprotease enzyme that cleaves and inhibits the activity of vWF. The presence of endothelial cell damage and dysregulation of the coagulation cascade is consistent with results in patients with critical disease or after death (3941). Our results not only demonstrate that these pathways become altered even in patients with early disease, but also provide the mechanistic link between plasma viremia and the hypercoagulable state observed in patients across the spectrum of COVID-19 disease severity $(2,42,43)$. These findings suggest that early interventions to prevent the circulatory dissemination of SARS-CoV-2 infection could help prevent these potentially devastating complications of COVID-19.

Interestingly, our proteomic analysis also showed a relationship between higher levels of viremia and persistent elevation of several SARS-CoV-2 entry factors, including FURIN and cathepsin $\mathrm{B} / \mathrm{L}$ (CTSB/CTSL). These results are consistent with a proteomic analysis of COVID-19 autopsies (39) in which Nie and colleagues reported that CTSB and CTSL, which are proteases that facilitate viral entry, are prominently elevated in the lungs. In contrast, ACE2, the primary host receptor for SARS-CoV-2, showed minimal upregulation (39). Cathepsin family proteins are also known for their role in facilitating SARS-CoV-2 spike protein priming as well as promoting the inflammasome/pyroptosis pathway, as the autopsy studies also reveal that inflammasome/pyroptosis-related proteins, including lactate dehydrogenase and MPO, are highly upregulated in lung tissues (39). The combination of our results and these autopsy findings points to the crucial role of the cathep$\sin$ family proteins in the pathogenesis of SARS-CoV-2.

Notably, the viremic and aviremic groups had comparable neutralization activity in this cohort. Most patients presented fairly early during their symptom course, and it is possible that neutralizing antibody titers were not yet particularly high or effective. More robust neutralizing antibody levels were detected during the course 
of hospitalization in all participants, regardless of the initial level of plasma viremia. It could also be that the level of plasma viremia is a reflection of the extent of tissue-based infection and less a reflection of the current level of neutralizing antibody titers. Our findings are consistent with several recent studies. For instance, Rydyznski Moderbacher et al. demonstrated that antibody level has only a moderate correlation with peak disease severity (44). Furthermore, the level and function (e.g., Fc $\gamma \mathrm{R}$ binding) of anti-SARS-CoV-2 RBD antibody are very similar between people with moderate and severe disease during early ( $<7$ days after symptom onset) and late (>14 days after symptom onset) stages of COVID-19, and diverge only between 7 and 14 days (45). In addition, 2 studies focused on SARS-CoV-2 viremia have demonstrated that levels of viremia were not associated with the neutralizing antibody level $(36,46)$. Furthermore, levels of neutralizing antibody titers were also not significantly correlated with levels of respiratory viral shedding (47). The interaction between SARS-CoV-2 antibody levels and viral load dynamics merits further investigation.

Our study also has a few notable limitations. Although quite comprehensive, our proteomic database does not cover all the cytokines and proteins of interest in COVID-19 pathogenesis. We rely on a preexisting proteomic database (17) and peripheral blood databases $(26,28)$ to infer the origin of differentially expressed proteins, but do not have data on single-cell RNA-Seq from this cohort to confirm the cellular source of some differentially expressed proteins. Given the relatively high limits of detection of culture-based assays, we are unable to confirm whether the RNA detected in plasma samples is from viable, infective SARS-CoV-2 virions; since plasma sample volume was limited, further subgenomic viral load analysis could not be performed. Additional studies will be needed to confirm the causal relationship between viremia and proteomic changes.

In summary, we report the largest study to date to demonstrate that SARS-CoV-2 viremia predicts severe COVID-19 disease outcomes and that systemic viral dissemination likely plays a role in mediating tissue damage, tissue fibrosis, hypercoagulable state, persistent elevation of proinflammatory markers, and higher viral entry factor expression. Our findings provide key insights into SARS-CoV-2 pathogenesis and identify potential therapeutic targets to mitigate COVID-19 disease severity.

\section{Methods}

Further information can be found in Supplemental Methods.

Study participants. Participant enrollment was described in our prior report (15). Briefly, participants were enrolled in the Emergency Department (ED) of Massachusetts General Hospital from March 24, 2020, to April 30, 2020, during the first peak of the COVID-19 surge. Symptomatic participants of 18 years or older with SARS-CoV-2 infection confirmed by nucleic acid tests were included in this current study. Clinical course was followed to 28 days after enrollment, or until hospital discharge if that occurred after 28 days.

Enrolled participants who were SARS-CoV-2 positive $(n=306)$ were categorized into 5 outcome/acuity groups: A1, death within 28 days; A2, requiring mechanical ventilation and survival to 28 days; A3, requiring hospitalization on supplemental oxygen within 28 days; A4, requiring hospitalization without need of supplemental oxygen; and A5, discharge from ED and no subsequent requirement of hospi- talization within 28 days. Severe disease was defined as belonging to group A1 or A2. In this current analysis, we included only participants with available plasma SARS-CoV-2 viral load $(n=300)$. Demographic classifications were made based on participants' report/response in medical record.

Study endpoints. The primary endpoint of this study was severe COVID-19 within 28 days of enrollment (intubation and/or death). Secondary endpoints included 28-day mortality and SARS-CoV-2 viremia.

Plasma SARS-CoV-2 viral load. Plasma SARS-CoV-2 viral load measurement was as reported in our previous study (11) with the following modifications. Briefly, RNA was extracted from $300 \mu \mathrm{L}$ of RPMI 1640-diluted EDTA-preserved plasma sample (RPMI 1640/plasma 2:1 dilution) (15) using a TRIzol-based method (Thermo Fisher Scientific). SARS-CoV-2 viral load was quantified using the US CDC 2019-nCoV_N1 primers and probe set (11). The lower limit of SARS-CoV-2 N gene quantification was 100 copies $/ \mathrm{mL}$. Samples with a positive signal but viral load lower than 100 copies/mL were denoted as detectable but unquantifiable.

Olink proteomic analyses. Proteomic analyses were described in a prior report (15). Briefly, the Olink Proximity Extension Assay (PEA) is a technology developed for high-multiplex analysis of proteins. Oligonucleotide-labeled monoclonal or polyclonal antibodies (PEA probes) are used to bind target proteins in a pairwise manner, thereby preventing all cross-reactive events. Upon binding, the oligonucleotides come in close proximity and hybridize, followed by extension, generating a unique barcode for identification. The full Olink library contains 1472 proteins and 48 control assays, dividing into inflammation, oncology, cardiometabolic, and neurology panels, with overlap in IL-6, IL-8/ CXCL8, and TNF- $\alpha$ for quality control (QC) purposes (Supplemental Figure 14 demonstrates the correlation of quadruplicate levels from each panel). Levels of proteins were denoted as normalized protein expression (NPX) units through a QC and normalization process developed and provided by Olink. Data generation of NPX consists of normalization to the extension control (known standard), $\log _{2}$-transformation, and level adjustment using the plate control (plasma sample). Information regarding protein expression at the tissue and blood cell levels, protein function, and protein localization was derived from the Human Protein Atlas $(48,49)$.

SARS-CoV-2 S pseudotyped lentivirus generation. Neutralizing antibody level was evaluated by pseudotyped lentivirus neutralization assay as reported in our recent study (15). Lentivirus vector was constructed using PCR amplification (Q5 High-Fidelity 2X Master Mix, New England Biolabs) from pUC57-nCoV-S (a gift from Jonathan Abraham, Blavatnik Institute, Harvard Medical School, Boston, Massachusetts, USA), in which the C-terminal 27 amino acids of SARSCoV-2 $\mathrm{S}$ are replaced by the NRVRQGYS sequence of HIV-1 (50). The truncated SARS-CoV-2 S fused to gp 41 was cloned into pCMV by Gibson assembly to obtain pCMV-SARS2 $\triangle \mathrm{C}$-gp41. Other vectors, including psPAX2, pCMV-VSV-G, pTRIP-SFFV-EGFPNLS (Addgene plasmid 86677), and pTRIP-SFFV-Hygro-2A-TMPRSS2, were described in our recent publication (15). The 293T ACE2/TMPRSS2 cell line was generated as described in our recent publication (15). 293T cells were seeded at $0.8 \times 10^{6}$ cells per well in a 6-well plate and were transfected the same day with a mix of DNA containing $1 \mu \mathrm{g}$ psPAX, $1.6 \mu \mathrm{g}$ pTRIP-SFFV-EGFP-NLS, and $0.4 \mu \mathrm{g}$ pCMV-SARS2 $\triangle \mathrm{C}$-gp41 using TransIT-293 Transfection Reagent (Mirus Bio). After overnight incubation, the medium was changed. SARS-CoV-2 $\mathrm{S}$ pseudotyped lentiviral particles were collected 30-34 hours after medium exchange and 
filtered using a $0.45 \mu \mathrm{m}$ syringe filter. To transduce 293T ACE2 cells, the same protocol was followed, with a mix containing $1 \mu \mathrm{g}$ psPAX, 1.6 $\mu \mathrm{g}$ pTRIP-SFFV-Hygro-2A-TMPRSS2, and $0.4 \mu \mathrm{g}$ pCMV-VSV-G.

SARS-CoV-2 S pseudotyped lentivirus antibody neutralization assay. 293T ACE2 cells have been widely used in other pseudotype neutralization assays $(51,52)$. One day before the neutralization experiment, 293T ACE2/TMPRSS2 cells were seeded at $5 \times 10^{3}$ cells in $100 \mu \mathrm{L}$ per well in 96-well plates. On the day of lentiviral harvest, $100 \mu \mathrm{L}$ SARSCoV-2 S pseudotyped lentivirus was incubated with $50 \mu \mathrm{L}$ of plasma diluted in medium to a final concentration of 1:100. Medium was then removed from 293T ACE2/TMPRSS2 cells and replaced with $150 \mu \mathrm{L}$ of the mix of plasma and pseudotyped lentivirus. Wells in the outermost rows of the 96-well plate were excluded from the assay. After overnight incubation, medium was changed to $100 \mu \mathrm{L}$ of fresh medium. Cells were harvested 40-44 hours after infection with TrypLE (Thermo Fisher Scientific), washed in medium, and fixed in FACS buffer containing 1\% PFA (Electron Microscopy Sciences). Percentage GFP was quantified on a Cytoflex LX flow cytometer (Beckman Coulter), and data were analyzed with FlowJo (Becton Dickinson). The events recorded at the flow cytometer do not suggest cell loss due to detachment. Neutralization rate was defined as 1 - (GFP\% $\left.\%_{\text {pseudovirustplasma }} / \mathrm{GFP} \%_{\text {pseudovirus alone }}\right)$.

Statistics. We summarized continuous variables using median and interquartile ranges (IQRs). For clinical variables, we used Wilcoxon's rank-sum test to compare continuous variables from 2 different categorical groups and Dunn's test for 3 or more groups. Categorical variables were evaluated using the $\chi^{2}$ test or Fisher's exact test. We used Spearman's rank correlation coefficient to evaluate correlation between different continuous variables. To evaluate the association of plasma SARS-CoV-2 viral load and clinical outcomes, we used logistic regression analyses to calculate odds ratios and $95 \%$ confidence intervals. Both univariate and multivariate logistic regression analyses were performed. In multivariate analyses, factors with a $P$ value less than 0.10 from univariate models were included. We also used Cox's proportional model to evaluate the correlation between viremia and 28-day mortality by calculating the hazard ratio. Clinical data analyses, logistic regression, and Cox proportion regression were performed on Stata (version 13.1), and figures were generated by Stata and GraphPad (Prism, version 9.0). $\mathrm{R}$ (version 4.0.2) was used to analyze proteomic data.

Linear regression models were fit independently to each protein using the lm package in $\mathrm{R}$ with protein values (NPX for Olink data) as the dependent variable. The models included a term for viremia and covariates for age, sex, ethnicity, heart disease, diabetes, hypertension, hyperlipidemia, pulmonary disease, kidney disease, and immunocompromised status to control for any potential confounding. $P$ values were adjusted to control the false discovery rate at $5 \%$ using the Benjamini-Hochberg method implemented in the emmeans package in $\mathrm{R}$.

Linear mixed-effects models were fit independently to each protein using the lme 4 package in R with protein values (NPX for Olink data) as the dependent variable. The model for viremia included a main effect of time, a main effect of viremia, the interaction between these 2 terms, and a random effect of patient ID to account for the correlation between samples coming from the same patient. Covariates for age, sex, ethnicity, heart disease, diabetes, hypertension, hyperlipidemia, pulmonary disease, kidney disease, and immunocompromised status were included in the model to control for any potential confounding effects. Details were reported in our recent study (15).

Study approval. This study was approved by the Mass General Brigham (formerly known as Partners) Institutional Review Board (IRB), with an IRB-approved waiver of informed consent (protocol 2017P001681).

\section{Author contributions}

JZL and YL conceptualized the study. MRF, BAP, ACV, MSF, NH, and MBG established the Massachusetts General Hospital ED cohort. MRF, NH, and MBG provided resources. YL, JR, and JPF performed the SARS-CoV-2 viral load assay. MG performed the neutralization assay. NCC, ALKG, IG, HKK, TJL, KMLP, BML, CLL, KM, JDM, BNM, BAP, MRL, BCR, NS, JT, and MFT performed sample collection and processing. YL, AMS, and AM performed formal analysis. KRK maintained the study database and oversaw study coordination. YL wrote the original draft. JZL, BAP, MRF, AMS, AM, $\mathrm{NH}, \mathrm{MBG}$, JR, and JPF reviewed and edited the draft.

\section{Acknowledgments}

We thank all the participants in this study. We thank all the clinical staff who made sample collection possible. Direct funding for this project was provided in part by a grant from Mark and Lisa Schwartz (to JZL), the NIH (U19AI082630 to NH), an American Lung Association COVID-19 Action Initiative grant (to MBG), and grants from the Executive Committee on Research at Massachusetts General Hospital (to MBG and MRF) and the Chan Zuckerberg Initiative (to ACV). NH was also funded by a gift from Arthur, Sandra, and Sarah Irving for the David P. Ryan, MD, Endowed Chair in Cancer Research. MG is the recipient of an EMBO Long-Term Fellowship (ALTF 486-2018) and a Cancer Research Institute/Bristol Myers Squibb Fellowship (CRI2993). This work was also supported by the Harvard Catalyst/Harvard Clinical and Translational Science Center (National Center for Advancing Translational Sciences, NIH awards UL1TR001102 and UL1TR002541-01) and by the Harvard University Center for AIDS Research (National Institute of Allergy and Infectious Diseases, 5P30AI060354). We are also very grateful for the generous contributions of Olink Proteomics Inc. and Novartis (in collaboration with SomaLogic Inc.), which provided in-kind all proteomics assays presented in this work, without which our findings would not have been possible.

Address correspondence to: Jonathan Z. Li, Brigham and Women's Hospital, Harvard Medical School, 65 Landsdowne Street, Room 421, Cambridge, Massachusetts 02139, USA. Phone: 617.768.8476; Email: jli@bwh.harvard.edu.
1. Johns Hopkins University \& Medicine. Johns Hopkins Coronavirus Resource Center. https:// coronavirus.jhu.edu/. Updated May 7, 2021. Accessed May 7, 2021.

2. Wiersinga WJ, et al. Pathophysiology, transmission, diagnosis, and treatment of Coronavirus disease 2019 (COVID-19): a review. JAMA 2020;324(8):782-793.

3. Li Y, et al. Liver fibrosis index FIB-4 is associated with mortality in COVID-19. Hepatol Commun. 2021;5(3):434-445.

4. Siddiqi HK, et al. Increased prevalence of myo- cardial injury in patients with SARS-CoV-2 viremia. Am JMed. 2021;134(4):542-546.

5. Choi B, et al. Persistence and evolution of SARS-

CoV-2 in an immunocompromised host. N EnglJ Med. 2020;383(23):2291-2293.

6. Puelles VG, et al. Multiorgan and renal 
tropism of SARS-CoV-2. N Engl J Med. 2020;383(6):590-592.

7. Ackermann M, et al. Pulmonary vascular endothelialitis, thrombosis, and angiogenesis in Covid-19. N Engl J Med. 2020;383(2):120-128.

8. Lindner D, et al. Association of cardiac infection with SARS-CoV-2 in confirmed COVID-19 autopsy cases. JAMA Cardiol. 2020;5(11):1281-1285.

9. Hanley B, et al. Histopathological findings and viral tropism in UK patients with severe fatal COVID-19: a post-mortem study. Lancet Microbe. 2020;1(6):e245-e253.

10. Vivanti AJ, et al. Transplacental transmission of SARS-CoV-2 infection. Nat Commun. 2020;11(1):3572.

11. Fajnzylber J, et al. SARS-CoV-2 viral load is associated with increased disease severity and mortality. Nat Commun. 2020;11(1):5493.

12. Hogan CA, et al. High frequency of SARS-CoV-2 RNAemia and association with severe disease. Clin Infect Dis. 2021;72(9):e291-e295.

13. Xu D, et al. Relationship between serum severe acute respiratory syndrome coronavirus 2 nucleic acid and organ damage in coronavirus 2019 patients: a cohort study [published online July 28, 2020]. Clin Infect Dis. https://doi. org/10.1093/cid/ciaa1085.

14. Chen X, et al. Detectable serum severe acute respiratory syndrome Coronavirus 2 viral load (RNAemia) is closely correlated with drastically elevated interleukin 6 level in critically ill patients with Coronavirus disease 2019. Clin Infect Dis. 2020;71(8):1937-1942.

15. Filbin MR, et al. Longitudinal proteomic analysis of severe COVID-19 reveals survival-associated signatures, tissue-specific cell death, and cell-cell interactions. Cell Rep Med. 2021; 2(5):100287.

16. Li Y, et al. Extracellular Nampt promotes macrophage survival via a nonenzymatic interleukin-6/STAT3 signaling mechanism. J Biol Chem. 2008;283(50):34833-34843.

17. Jiang $\mathrm{L}$, et al. A quantitative proteome map of the human body. Cell. 2020;183(1):269-283.

18. Wrapp D, et al. Cryo-EM structure of the 2019$\mathrm{nCoV}$ spike in the prefusion conformation. Science. 2020;367(6483):1260-1263.

19. Amraie R, et al. CD209L/L-SIGN and CD209/ DC-SIGN act as receptors for SARS-CoV-2 and are differentially expressed in lung and kidney epithelial and endothelial cells [preprint]. https:// doi.org/10.1101/2020.06.22.165803. Posted on bioRxiv June 23, 2020.

20. Cantuti-Castelvetri L, et al. Neuropilin-1 facilitates SARS-CoV-2 cell entry and infectivity. Science. 2020;370(6518):856-860.

21. Daly JL, et al. Neuropilin- 1 is a host factor for SARS-CoV-2 infection. Science. 2020;370(6518):861-865.

22. Hoffmann M, et al. A multibasic cleavage site in the spike protein of SARS-CoV-2 is essential for infection of human lung cells. Mol Cell. 2020;78(4):779-784.

23. Hoffmann M, et al. SARS-CoV-2 cell entry depends on ACE2 and TMPRSS2 and is blocked by a clinically proven protease inhibitor. Cell. 2020;181(2):271-280.

24. Rayamajhi M, et al. Detection of pyroptosis by measuring released lactate dehydrogenase activity. Methods Mol Biol. 2013;1040:85-90.

25. Lee JS, et al. Immunophenotyping of COVID-19 and influenza highlights the role of type I interferons in development of severe COVID-19. Sci Immunol. 2020;5(49):eabd1554.

26. Wilk AJ, et al. A single-cell atlas of the peripheral immune response in patients with severe COVID-19. Nat Med. 2020;26(7):1070-1076.

27. Schulte-Schrepping J, et al. Severe COVID-19 is marked by a dysregulated myeloid cell compartment. Cell. 2020;182(6):1419-1440.

28. Monaco G, et al. RNA-Seq signatures normalized by mRNA abundance allow absolute deconvolution of human immune cell types. Cell Rep. 2019;26(6):1627-1640.

29. He YW, et al. The extracellular matrix protein mindin is a pattern-recognition molecule for microbial pathogens. Nat Immunol. 2004;5(1):88-97.

30. Koduri RS, et al. Bactericidal properties of human and murine groups I, II, V, X, and XII secreted phospholipases A(2). J Biol Chem. 2002;277(8):5849-5857.

31. Bastard P, et al. Autoantibodies against type I IFNs in patients with life-threatening COVID-19. Science. 2020;370(6515):eabd4585.

32. Grant PR, et al. Detection of SARS coronavirus in plasma by real-time RT-PCR. $N$ Engl J Med. 2003;349(25):2468-2469.

33. Choi SM, et al. Influenza viral RNA detection in blood as a marker to predict disease severity in hematopoietic cell transplant recipients. J Infect Dis. 2012;206(12):1872-1877.

34. Waghmare A, et al. Respiratory syncytial virus lower respiratory disease in hematopoietic cell transplant recipients: viral RNA detection in blood, antiviral treatment, and clinical outcomes. Clin Infect Dis. 2013;57(12):1731-1741.

35. Taniguchi $\mathrm{K}$, et al. Incidence and treatment strategy for disseminated adenovirus disease after haploidentical stem cell transplantation. Ann Hematol. 2012;91(8):1305-1312.

36. Prebensen C, et al. SARS-CoV-2 RNA in plasma is associated with ICU admission and mortality in patients hospitalized with COVID-19 [published online September 5, 2020]. Clin Infect Dis. https://doi.org/10.1093/cid/ciaa1338.

37. Bermejo-Martin JF, et al. Viral RNA load in plasma is associated with critical illness and a dysregulated host response in COVID-19. Crit Care.
2020;24(1):691.

38. Berastegui-Cabrera J, et al. SARS-CoV-2 RNAemia is associated with severe chronic underlying diseases but not with nasopharyngeal viral load. JInfect. 2021;82(3):e38-e41.

39. Nie X, et al. Multi-organ proteomic landscape of COVID-19 autopsies. Cell. 2021;184(3):775-791.

40. Gutmann C, et al. SARS-CoV-2 RNAemia and proteomic trajectories inform prognostication in COVID-19 patients admitted to intensive care. Nat Commun. 2021;12(1):3406.

41. Delrue M, et al. von Willebrand factor/ ADAMTS13 axis and venous thromboembolism in moderate-to-severe COVID-19 patients. $\mathrm{Br} J$ Haematol. 2021;192(6):1097-1100.

42. Wichmann D, et al. Autopsy findings and venous thromboembolism in patients with COVID-19: a prospective cohort study. Ann Intern Med. 2020;173(4):268-277.

43. Oxley TJ, et al. Large-vessel stroke as a presenting feature of Covid-19 in the young. $N$ Engl J Med. 2020;382(20):e60.

44. Rydyznski Moderbacher C, et al. Antigen-specific adaptive immunity to SARS-CoV- 2 in acute COVID-19 and associations with age and disease severity. Cell. 2020;183(4):996-1012.

45. Zohar T, et al. Compromised humoral functional evolution tracks with SARS-CoV-2 mortality. Cell. 2020;183(6):1508-1519.

46. Jacobs JL, et al. COVID-19 outcome: insights from quantification of viremia and neutralizing antibody. Paper presented at: Conference on Retroviruses and Opportunistic Infections; March 6-10, 2021; virtual. https://www. croiconference.org/abstract/covid-19outcome-insights-from-quantification-ofviremia-and-neutralizing-antibody/. Accessed May 7, 2021.

47. Wang Y, et al. Kinetics of viral load and antibody response in relation to COVID-19 severity. JClin Invest. 2020;130(10):5235-5244.

48. Uhlén M, et al. Proteomics. Tissue-based map of the human proteome. Science. 2015;347(6220):1260419.

49. Uhlen M, et al. A genome-wide transcriptomic analysis of protein-coding genes in human blood cells. Science. 2019;366(6472): eaax9198.

50. Moore MJ, et al. Retroviruses pseudotyped with the severe acute respiratory syndrome coronavirus spike protein efficiently infect cells expressing angiotensin-converting enzyme 2.J Virol. 2004;78(19):10628-10635.

51. Schmidt F, et al. Measuring SARS-CoV-2 neutralizing antibody activity using pseudotyped and chimeric viruses. J Exp Med. 2020;217(11):e20201181.

52. Robbiani DF, et al. Convergent antibody responses to SARS-CoV-2 in convalescent individuals. Nature. 2020;584(7821):437-442. 